\title{
Severe recurrent nocturnal hypoglycemia during chemotherapy with 6-mercaptopurine in a child with acute lymphoblastic leukemia
}

\author{
Eun Mi Cho, MD, \\ Jung Eun Moon, MD, \\ Soo Jung Lee, MD, \\ Cheol Woo Ko, MD, PhD
}

Department of Pediatric Endocrinology, Kyungpook National University School of Medicine, Kyungpook National University Children's Hospital, Daegu, Korea
Received: 3 January, 2018

Revised: 18 February, 2018

Accepted: 18 March, 2018

Address for correspondence:

Cheol Woo Ko, MD, PhD

Department of Pediatric Endocrinology, Kyungpook National University Children's Hospital, 807, Hoguk-ro, Buk-gu, Daegu 41404, Korea

Tel: +82-53-420-5715

Fax: +82-53-425-6683

E-mail: cwko@knu.ac.kr

https://orcid.org/0000-0002-06437233
Various endocrine dysfunctions occur during chemotherapy, including hypoglycemia. However, reports of hypoglycemia associated with 6-mercaptopurine (6-MP) are rare. Herein, we report an 8-year-old boy with severe symptomatic hypoglycemia likely due to 6-MP during chemotherapy. He had been diagnosed with acute lymphoblastic leukemia 3 years previously and was in the maintenance chemotherapy period. Treatment included oral dexamethasone, methotrexate, and 6-MP, of which only 6-MP was administered daily. Hypoglycemic symptoms appeared mainly at dawn, and his serum glucose dropped to a minimum of $37 \mathrm{mg} / \mathrm{dL}$. Laboratory findings showed nothing specific other than increased serum cortisol, free fatty acids, ketone, alanine aminotransferase, and aspartate aminotransferase. Under the hypothesis of hypoglycemia due to chemotherapy drugs, we changed the time of 6-MP from evening to morning and recommended him to ingest carbohydrate-rich foods before bedtime. Hypoglycemia improved dramatically, and there was no further episode during the remaining maintenance chemotherapy period. To the best of our knowledge, this is the first report of this type of hypoglycemia occurring in an Asian child including Korean.

Keywords: Hypoglycemia, 6-Mercaptopurine, Acute lymphoblastic leukemia, Maintenance chemotherapy

\section{Introduction}

Definition of hypoglycemia as an exact value is controversial. Clinically, hypoglycemia may be defined as a low serum glucose level that causes brain dysfunction. ${ }^{1)}$ Hypoglycemia may sometimes be caused by the adverse effects of drugs. ${ }^{2}$ Many drugs are used for acute lymphoblastic leukemia (ALL) chemotherapy, and 6-mercaptopurine (6-MP) is the main drug of maintenance chemotherapy. Although mild hypoglycemia is a common adverse effect of chemotherapy, severe hypoglycemia is rare. ${ }^{3)}$ We report the case of a child with ALL who developed severe nocturnal hypoglycemia during maintenance chemotherapy, possibly caused by the oral administration of 6-MP. To the best of our knowledge, there has been no previous report of severe hypoglycemia associated with 6-MP in Asia including Korea.

\section{Case report}

An 8-year-old obese boy was diagnosed with ALL L2 standard risk group 3 years ago and was currently receiving maintenance chemotherapy according to the COG AALL0331 protocol (COG, Children's Oncology Group-the world's largest organization devoted exclusively to pediatric cancer research. AALL0331 is the protocol for standard risk ALL.). The 
treatment included the oral administration of dexamethasone $(3$ $\mathrm{mg} / \mathrm{m}^{2}$ per dose twice daily for 5 days every 4 weeks), 6-MP (75 $\mathrm{mg} / \mathrm{m}^{2}$ per dose daily), and methotrexate $\left(20 \mathrm{mg} / \mathrm{m}^{2}\right.$ per dose per week). The patient complained of chest discomfort, fatigue, and cold sweating at dawn for several months. These symptoms mainly occurred on the day when he ate dinner early and did not eat anything before he fell asleep. After admission, he ate a regular meal, and we checked his blood glucose continuously (Fig. 1). Symptoms such as dizziness, chest discomfort, nausea, drowsy mentality and even eyeball deviation were constantly observed at dawn or in the early morning. When these symptoms were present, his serum glucose level dropped to a minimum of $37 \mathrm{mg} / \mathrm{dL}$. After intravenous injection of $20 \%$ dextrose solution $(1 \mathrm{~mL} / \mathrm{kg})$, these symptoms resolved. His blood cell counts and electrolytes were within the normal range, but his serum aspartate aminotransferases (AST) and alanine aminotransferase (ALT) levels were elevated. Fasting serum insulin and C-peptide levels were elevated, and serum and urine ketones were positive (Table 1). The glucagon stimulation test was performed for the differential diagnosis between hyperinsulinemic hypoglycemia and ketotic hypoglycemia, and the result was negative (Table 2). We suspected secondary hypoglycemia due to chemotherapy drugs; according to the references, 6-MP was a possible cause of hypoglycemia. We switched the administration time of 6-MP from evening to morning and recommended the ingestion of carbohydrate-rich

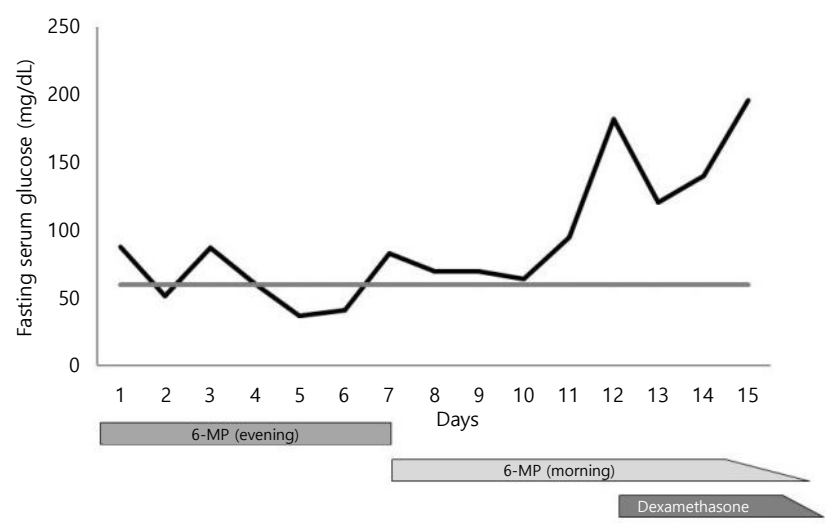

Fig. 1. This figure shows the change in fasting serum glucose level checked early morning during hospitalization. On the 7th day of admission, the dosing time of 6-mercaptopurine (6-MP) was changed to morning, and no further hypoglycemic episodes were observed. After 12 days of admission, oral dexamethasone was started according to the COG AALL 0331 protocol, and fasting serum glucose level showed a sharp increase (horizontal gray line=fasting serum glucose 60). COG, Children's Oncology Group-the world's largest organization devoted exclusively to pediatric cancer research; AALL0331, protocol for standard risk acute lymphoblastic leukemia. foods before bedtime. Since then, the number of hypoglycemic episodes decreased significantly. He was discharged, and the hypoglycemic symptoms were no longer present.

This study was approved by the Institutional Review Board of Kyungpook National University Children's Hospital (approval number: KNUCH 2017-12-008). Informed consent was waived by the IRB.

\section{Discussion}

Endocrine dysfunctions associated with ALL chemotherapy occurs frequently both during and after therapy and even many years later. ${ }^{4}$ The most common cause of hypoglycemia in children with ALL is the use of excessive amounts of insulin for the treatment of hyperglycemia. Meanwhile, it has been reported that hypoglycemia can also occur in children receiving maintenance chemotherapy with 6-MP. ${ }^{3,5)}$

Symptomatic hypoglycemia due to 6-MP is one of its rare side effects, and only a few cases have been reported. El-Bitar et al. ${ }^{6}$ reported prolonged generalized tonic nocturnal seizures and severe hypoglycemia in a 4-year-old boy who was diagnosed with ALL and received 6-MP as maintenance therapy. The symptoms occurred after taking the evening dose of 6-MP after a 12 -hour period of fasting. Bay et al. ${ }^{7)}$ reported the occurrence of symptomatic hypoglycemia in children with pre-B cell ALL, in which case symptoms occurred the following morning after taking 6-MP in the evening. Visavachaipan et al. ${ }^{8)}$ reported that a 5-year-old obese boy who was receiving 6-MP maintenance chemotherapy for ALL developed symptomatic hypoglycemia

Table 1. Clinical and laboratory characteristics in study patient at admission

\begin{tabular}{lcc}
\hline Characteristic & Value & Reference range \\
\hline Age $(\mathrm{yr})$ & 8.2 & - \\
Sex & Male & - \\
Height $(\mathrm{cm})$ & 124.3 (50\%ile) & - \\
Weight $(\mathrm{kg})$ & 33.9 (90\%ile) & - \\
Body mass index $\left(\mathrm{kg} / \mathrm{m}^{2}\right)$ & $22.0(>95 \%$ ile) & - \\
Fasting glucose $(\mathrm{mg} / \mathrm{dL})$ & 88 & $60-100$ \\
Fasting insulin $(\mu \mathrm{U} / \mathrm{mL})$ & 16.7 & $<2-13$ \\
Fasting C-peptide $(\mathrm{ng} / \mathrm{mL})$ & 2.4 & $0.4-2.2$ \\
Cortisol $(\mu \mathrm{g} / \mathrm{dL})$ & 42.1 & $3-21$ \\
AST $(\mathrm{U} / \mathrm{L})$ & 126 & $3-45$ \\
ALT $(\mathrm{U} / \mathrm{L})$ & 232 & $3-45$ \\
Serum ketone $(\mu \mathrm{mol} / \mathrm{L})$ & $1,126.8$ & $<160$ \\
\hline
\end{tabular}

BMI, body mass index; AST, aspartate aminotransferase; ALT, alanine aminotransferase.

*Cortisol was checked via serum sampled early in the morning.

Table 2. Results of glucagon stimulation test

\begin{tabular}{lccccccc}
\hline & \multicolumn{7}{c}{ Time $(\mathrm{min})$} \\
\hline
\end{tabular}


that occurred after 8-10 hours of fasting. However, there has been no previous report of hypoglycemia associated with 6-MP in Asian children.

Although 6-MP is very commonly used in ALL maintenance chemotherapy, little is known about the mechanism of 6-MP-induced hypoglycemia. Halonen et al. ${ }^{3)}$ reported that gluconeogenic amino acid and free carnitine serum levels were reduced in the fasting state with maintenance chemotherapy in children with ALL. Decreased gluconeogenic substrate was described as the main mechanism of hypoglycemia. Liu et al. ${ }^{9}$ confirmed that 6-MP activates nuclear receptor subfamily 4, group A, member 3 , resulting in increased glucose transport and glucose transporter type 4 translocation into the basal muscle cell. This mechanism may increase skeletal muscle glucose uptake, and hypoglycemia may occur regardless of the insulin level. In our patient, several abnormal laboratory findings were observed. First of all, his hypoglycemia was symptomatic, and the symptoms were restored when the hypoglycemia was solved. It is contented with Whipple's triad. Hyperinsulinemia is thought to be the result of insulin resistance due to obesity. Increased AST and ALT levels are also thought to be the result of fatty liver due to obesity or liver injury caused by hepatotoxic drugs such as 6-MP. Serum and urine ketone positivity are caused by impaired glucose metabolism due to fasting, which is likely to worsen the injury to the liver, and therefore hypoglycemia as well.

Symptoms of hypoglycemia in children may be nonspecific, ${ }^{10)}$ and unexpected hypoglycemia may occur during chemotherapy. To prevent hypoglycemia, we changed the dosing time of 6-MP to morning and recommended him to eat carbohydrate-rich food before bedtime. In most of the literature, this condition was treated with a similar approach, and hypoglycemia was found to be improved. ${ }^{3,5,11)}$ For children aged under 6 years in particular, it is recommended to wake up and drink milk or juice after 8 to 9 hours of sleep. ${ }^{6.8)}$ If hypoglycemic symptoms are very severe, the dose of 6-MP may be reduced for a short period of time. ${ }^{7)}$ To prevent hypoglycemia due to 6-MP, clinicians should be aware that 6-MP can cause hypoglycemia and the possibility of hypoglycemia during maintenance chemotherapy should be carefully monitored. And, if necessary, the chemotherapy protocol may be revised. Further studies on the mechanism of hypoglycemia due to 6-MP and its appropriate treatment strategies are necessary.

\section{Conflict of interest}

No potential conflict of interest relevant to this article was reported.

\section{References}

1. Thornton PS, Stanley CA, De Leon DD, Harris D, Haymond MW, Hussain K, et al. Recommendations from the Pediatric Endocrine Society for Evaluation and Management of Persistent Hypoglycemia in Neonates, Infants, and Children. J Pediatr 2015;167:238-45.

2. Ben Salem C, Fathallah N, Hmouda H, Bouraoui K. Druginduced hypoglycaemia: an update. Drug Saf 2011;34:2145.

3. Halonen P, Salo MK, Mäkipernaa A. Fasting hypoglycemia is common during maintenance therapy for childhood acute lymphoblastic leukemia. J Pediatr 2001;138:428-31.

4. Howard SC, Pui CH. Endocrine complications in pediatric patients with acute lymphoblastic leukemia. Blood Rev 2002;16:225-43.

5. Ziino O, Russo D, Orlando MA, Benigno V, Locatelli F, Aricò M. Symptomatic hypoglycemia in children receiving oral purine analogues for treatment of childhood acute lymphoblastic leukemia. Med Pediatr Oncol 2002;39:32-4.

6. El-Bitar MK, Muwakkit SA, Dabbagh O. Severe hypoglycemic seizures in a child receiving 6-mercaptopurine. J Pediatr Hematol Oncol 2011;33:e75-6.

7. Bay A, Oner AF, Cesur Y, Dogan M, Etlik O, Sanli F. Symptomatic hypoglycemia: an unusual side effect of oral purine analogues for treatment of ALL. Pediatr Blood Cancer 2006;47:330-1.

8. Visavachaipan N, Aledo A, Franklin BH, Brar PC. Continuous glucose monitoring: a valuable monitoring tool for management of hypoglycemia during chemotherapy for acute lymphoblastic leukemia. Diabetes Technol Ther 2013; 15:97-100.

9. Liu Q, Zhu X, Xu L, Fu Y, Garvey WT. 6-Mercaptopurine augments glucose transport activity in skeletal muscle cells in part via a mechanism dependent upon orphan nuclear receptor NR4A3. Am J Physiol Endocrinol Metab 2013;305:E1081-92.

10. Melachuri S, Gandrud L, Bostrom B. The association between fasting hypoglycemia and methylated mercaptopurine metabolites in children with acute lymphoblastic leukemia. Pediatr Blood Cancer 2014;61:1003-6.

11. Halonen P, Salo MK, Schmiegelow K, Mäkipernaa A. Investigation of the mechanisms of therapy-related hypoglycaemia in children with acute lymphoblastic leukaemia. Acta Paediatr 2003;92:37-42. 